\title{
Simultaneous Hard X-ray Ptychographic Tomography and X-ray Fluorescence To- mography of Isolated Hollow Core-Shell GaN Rods
}

\author{
$\underline{\text { M. Kahnt }}^{1,2 *}$, G. Falkenberg ${ }^{1}$, J. Garrevoet ${ }^{1}$, J. Hartmann ${ }^{4}$, T. Krause ${ }^{3}$, M. Niehle ${ }^{3}$, M. Scholz ${ }^{2}$, M. \\ Seyrich $^{1,2}$, A. Trampert ${ }^{3}$, A. Waag ${ }^{4}$, H.-H. Wehmann ${ }^{4}$, F. Wittwer ${ }^{1,2}$, H. Zhou ${ }^{4}$ M. Hanke ${ }^{3}$ and C. G. \\ Schroer ${ }^{1,2}$ \\ ${ }^{1}$ Deutsches Elektronen-Synchrotron, Hamburg, Germany \\ ${ }^{2}$ University of Hamburg, Department Physik, Hamburg, Germany \\ ${ }^{3}$ Paul-Drude-Institut für Festkörperelektronik, Berlin, Germany \\ ${ }^{4}$ Institut für Halbleitertechnik and Laboratory for Emerging Nanometrology, Technische Universität Braun- \\ schweig, Braunschweig, Germany \\ *Corresponding author: maik.kahnt@ desy.de
}

X-ray ptychographic tomography (XPCT) and X-ray fluorescence (XRF) tomography are two methods that utilize the same scanning scheme and have compatible measurement geometries. The differences can be found in the requirements for the probing beam. An XRF measurement relies on the probing beam to be small, while the XPCT measurement only relies on the probing beam to be coherent. A small noncoherent beam can be prepared with a significantly higher flux compared to a coherent one at current third generation synchrotron sources. Therefore these two kinds of measurements are usually done sequentially and not simultaneously.

In this work we discuss the advantages and drawbacks of such multimodal / simultaneous measurements on the example of a tomography experiment performed on core-shell structured micro-rods. These structures are of great interest for future electronic, optoelectronic or sensoric applications as they increase the active area dramatically compared to planar systems. Hence, analyzing and optimizing these structures has a great significance for industrial applications. The shell of the analysed micro-rods consists of an InGaN/GaN double layer. A red shift of the emitted light of the $\mathrm{InGaN}$ was observed performing spatially resolved cathodoluminescence (CL) measurements using an SEM [1]. The reason for this red shift could be a thickness and/or a concentration gradient in the center $(\mathrm{In} / \mathrm{Ga}) \mathrm{N}$ shell of the micro-rod. XPCT has the power to answer both questions due to it's high spatial resolving power of the sample's electron density. An XRF tomogram on the other hand has a higher elemental contrast, but an inferior spatial resolution. The sample, a single free

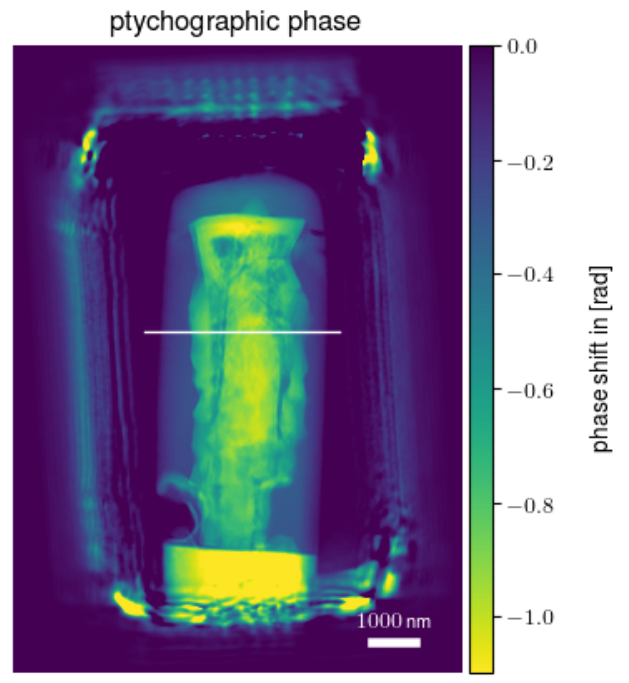

Figure 1: One of the 181 recorded projections. The thin horizontal white line marks the height of the slices shown in figure 2.

standing micro-rod, was prepared by fixing a group of micro-rods with an organic glue before a single rod was extracted using focused ion beam milling. The experiment was done at the hard X-ray nanoprobe beamline P06 (PETRA III, Hamburg, DE) [2]. Nano-focusing lenses were used to create a probing beam of $173 \mathrm{~nm}$ (horizontal) $\times 189 \mathrm{~nm}$ (vertical) at $10.47 \mathrm{keV}$ (just above the Ga- $K_{\alpha}$ edge) with a mean flux of $3 \times 10^{7} \mathrm{ph} \cdot \mathrm{s}^{-1}$. The projections consist of 23353 exposures with $50 \mathrm{~nm}$ scanning steps and were recorded at $1^{\circ}$ steps over an $180^{\circ}$ arc, resulting in a Nyquist resolution limit of $35 \mathrm{~nm}$ in the horizontal plane. Exposures were taken at $50 \mathrm{~Hz}$ and include one diffraction pattern for XPCT and one XRF spectrum. Thus the 
measuring time for one projection was $8 \mathrm{~min}$ and one day for a whole tomogram. The movement of the sample perpendicular to the X-ray beam was recorded using three interferometer beams retro-reflected by a ball lens below the sample [3].

Each of the 181 projections was using the 'ePie'-algorithm [4]. The elemental maps from the fitted XRF spectra were up-sampled to same pixel grid as the ptychographic reconstructions. Using the ptychographic phase images as a reference all images (ptychographic phase, ptychographic amplitude and the upsampled elemental maps) were aligned [5]. While relatively aligning projections between different rotation angles, the maps belonging to different contrasts but the same rotation angle were never shifted relative to each other, since they were recorded simultaneously and thus do not need a relative alignment. Afterwards the volumes were reconstructed slice by slice using a filtered back-projection algorithm from the tomopy framework [6]. A central slice is shown in figure 2 for the three major contrasts.
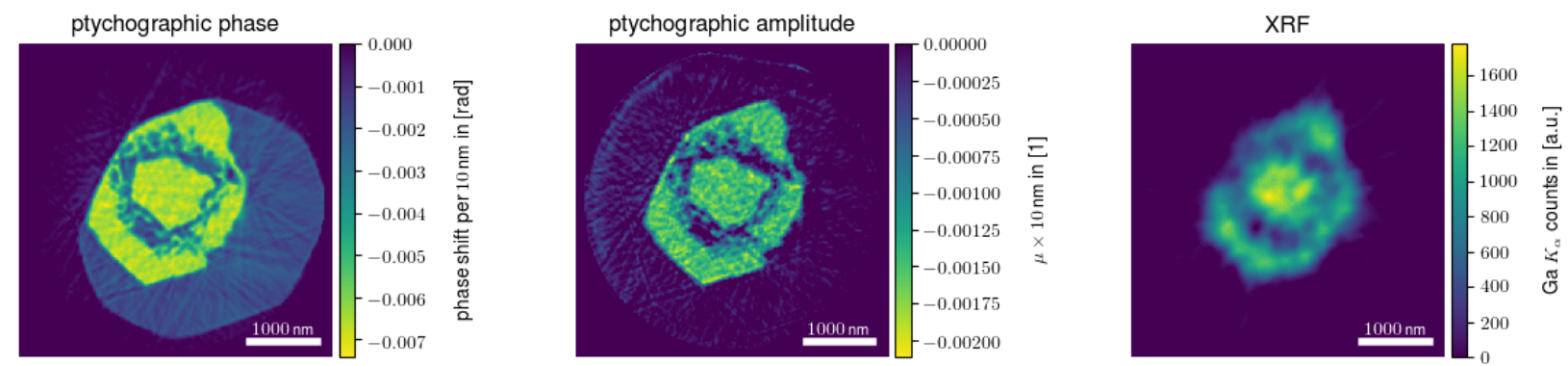

Figure 2: The same reconstructed tomographic slice for three different contrasts of the sample recorded at $10.47 \mathrm{keV}$. Left: the reconstructed ptychographic phase shift; Center: the reconstructed absorption, and Right: the reconstructed XRF photon yield for the $\mathrm{Ga}-\mathrm{K}_{\alpha}$ line.

The resolution of the volumes reconstructed from the XPCT data was calculated to $36.8 \mathrm{~nm}$ using Fourier shell correlation. This is just above the resolution limit given by the angular sampling and 5 times smaller than the beam size. Surprisingly the tested micro-rods did not have the first shell layer, consisting of (In/Ga)N. Instead the measured phase shift and absorption at the position of this shell matched the organic glue surrounding the micro rod. The current hypothesis is, that the organic glue dissolved the less stable first (In, Ga)N shell layer trough holes in the outermost GaN shell layer and then filled the void inside.

We have shown that these multimodal measurements can routinely be performed at current sources, allowing to reconstruct inherently aligned six (or higher)-dimensional datasets (three spatial dimensions, phase shift, absorption and Ga-XRF yield of the sample). The XPCT data had the expected superior resolution of $36.8 \mathrm{~nm}$ compared to the $\approx 180 \mathrm{~nm}$ of the XRF data. Reconstructing the projections for one tomographic dataset took about 500 GPU hours (Nvidia K40) due to the vast number of recorded diffraction patterns. This amount of data was expected when scanning a large sample with a small probing beam. At upcoming Diffraction Limited Storage Rings DLSRs the coherent flux will increase by two orders of magnitude. Therefore the reason to separate XPCT and XRF tomography measurements becomes void and these kinds of simultaneous measurements will be done routinely.

\section{References:}

[1] T. Krause, et al. Phys. Rev. Applied 7, 024033 (2017).

[2] C. G. Schroer, et al. Nucl. Instr. Meth. A, 616(2-3), 93-97 (2010).

[3] C. G. Schroer, et al. Proc. of SPIE 10389, 1038901 (2017).

[4] A. M. Maiden, et al. Ultramicroscopy 109, 1256-1262 (2009).

[5] M. Guizar-Sicairos, et al. Optics Express 19, 21345-21357 (2011).

[6] D. Gürsoy, et al. J Synchrotron Radiat. 21, 1188-1193. (2014). 